\title{
2-Fibonacci polynomials in the family of Fibonacci numbers
}

\author{
Engin Özkan ${ }^{1}$, Merve Taştan $^{1}$ and Ali Aydoğdu ${ }^{2}$ \\ ${ }^{1}$ Department of Mathematics, University of Erzincan Binali Yıldırım \\ Faculty of Arts and Sciences, Yalnızbağ Campus, 24100, Erzincan, Turkey \\ e-mails: eozkanmath@gmail.com, mervetastan24@gmail.com \\ ${ }^{2}$ Department of Mathematics, University of Beykent \\ Ayazağa Campus, Ayazağa- Maslak, Sarıyer, 34485, İstanbul, Turkey \\ e-mail: aydogduali84@gmail.com
}

Received: 3 April $2018 \quad$ Revised: 17 August $2018 \quad$ Accepted: 19 August 2018

\begin{abstract}
In the present study, we define new 2-Fibonacci polynomials by using terms of a new family of Fibonacci numbers given in [4]. We show that there is a relationship between the coefficient of the 2-Fibonacci polynomials and Pascal's triangle. We give some identities of the 2-Fibonacci polynomials. Afterwards, we compare the polynomials with known Fibonacci polynomials. We also express 2-Fibonacci polynomials by the Fibonacci polynomials. Furthermore, we prove some theorems related to the polynomials. Also, we introduce the derivative of the 2-Fibonacci polynomials.
\end{abstract}

Keywords: Fibonacci numbers, Fibonacci polynomials, Generalized Fibonacci polynomials. 2010 Mathematics Subject Classification: 11B39.

\section{Introduction}

Fibonacci numbers are of a great importance in mathematics and they have many important applications to diverse fields (see, e.g., [7]). The Fibonacci numbers $F_{n}$ are given by the recurrence relation

$$
F_{n+1}=F_{n}+F_{n-1}, \quad n \geq 1
$$


with the initial conditions $F_{0}=0$ and $F_{1}=1$. It is well known that the Fibonacci numbers are given by Binet's formula

$$
F_{n}=\frac{1}{\sqrt{5}}\left(\alpha^{n+1}-\beta^{n+1}\right), \quad n=0,1,2, \ldots,
$$

where $\alpha=(1+\sqrt{5}) / 2$ and $\beta=(1-\sqrt{5}) / 2$.

The Fibonacci polynomials were studied in 1883 by E. C. Catalan and E. Jacobsthal. The Fibonacci polynomials $F_{n}(x)$ studied by Catalan are defined by the recurrence relation

$$
F_{n+2}(x)=x F_{n+1}(x)+F_{n}(x), \quad n \geq 1
$$

with initial condition $F_{1}(x)=1, F_{2}(x)=x$. The polynomials $J_{n}(x)$ studied by Jacobsthal are defined by

$$
J_{n+2}(x)=J_{n+1}(x)+x J_{n}(x),
$$

where $J_{0}(x)=J_{1}(x)=1$ and $n \geq 0$.

It is well known that the Fibonacci polynomials bear relation with the Fibonacci numbers, where $F_{n}(1)=F_{n}$ is the $n$-th Fibonacci number. Some identities for the Fibonacci polynomials are given by Swamy [13]. Hoggatt and Lind [3] made a similar symbolic substitution of certain sequence into the Fibonacci polynomials. Hoggatt et al. [4] obtained some more identities for Fibonacci and Lucas Polynomials. Ivie [6] introduced a general type of Q-matrix for the generalized Fibonacci sequence. Hoggatt and Bicknell [5] generalized the Fibonacci polynomials and their relationship to diagonals of Pascal's triangle. Falcon and Plaza [2] presented the derivatives of $k$-Fibonacci polynomials as convolution of the $k$-Fibonacci polynomials and proved many relations for the derivatives of Fibonacci polynomials. Nalli and Haukkanen [10] introduced the $h(x)$-Fibonacci polynomials, where $h(x)$ is a polynomial with real coefficient. C. Berg [1] gave Fibonacci numbers and orthogonal polynomials. Tuğlu et al. [14] introduced generalized bivariate Fibonacci and Lucas p-polynomails. Lee and Asci [8] defined a new generalization of Fibonacci polynomials called $(p, q)$-Fibonacci polynomials and obtained combinatorial identities. Panwar et al. [11] described sums of generalized Fibonacci polynomials. Ramirez [12] introduced the convolved $h(x)$-Fibonacci polynomials. Ye and Zhang [15] gave a common generalization of convolved generalized Fibonacci and Lucas polynomials.

The paper is organized as follows. In Section 2, we briefly review some basic concepts about the $k$-Fibonacci numbers and Fibonacci polynomials. In Section 3, we define new 2-Fibonacci polynomials by using terms of a new family of Fibonacci numbers given in [9] and show that the relationship between Pascal's Triangle and the coefficients of the 2-Fibonacci polynomials. Also, we present 2-Fibonacci polynomials using the Fibonacci polynomials and give the derivatives of the 2-Fibonacci polynomials.

\section{Materials and methods}

It is well-known that the Fibonacci polynomials: $F_{n}(x)$ for $n=0,1,2, \ldots$ studied by Catalan are defined by the recurrence relation

$$
F_{n+2}(x)=x F_{n+1}(x)+F_{n}(x),
$$


where $F_{0}(x)=0, F_{1}(x)=1, F_{2}(x)=x$ and $n \geq 1$, the Fibonacci polynomials are generated by matrix

$$
Q_{2}=\left(\begin{array}{cc}
x & 1 \\
1 & 0
\end{array}\right), \quad Q_{2}^{n}=\left(\begin{array}{cc}
F_{n+1}(x) & F_{n}(x) \\
F_{n}(x) & F_{n-1}(x)
\end{array}\right) .
$$

This can be verified quite easily by mathematical induction. If $x=1$, then $F_{n}(1)$ is the $n^{\text {th }}$ Fibonacci number $F_{n}$, and if $x=2$, then $F_{n}(2)$ is the $n^{\text {th }}$ Pell number $P_{n}$.

If one writes Pascal's triangle in left-justified form, one obtains the Fibonacci numbers by adding the elements along the rising diagonals. Indeed, those elements are the coefficients of the Fibonacci polynomials. That is

$$
F_{n}(x)=\sum_{j=0}^{[(n-1) / 2]}\left(\begin{array}{c}
n-j-1 \\
j
\end{array}\right) x^{n-2 j-1}
$$

where $[x]$ is the greatest integer contained in $x$, and $\left(\begin{array}{l}n \\ j\end{array}\right)$ is a binomial coefficient.

\begin{tabular}{|c|c|}
\hline Fibonacci polynomials & Coefficients array \\
\hline$F_{1}(x)=1$ & 1 \\
\hline$F_{2}(x)=x$ & 1 \\
\hline$F_{3}(x)=x^{2}+1$ & 11 \\
\hline$F_{4}(x)=x^{3}+2 x$ & 12 \\
\hline$F_{5}(x)=x^{4}+3 x^{2}+1$ & $\begin{array}{lll}1 & 3 & 1\end{array}$ \\
\hline$F_{6}(x)=x^{5}+4 x^{3}+3 x$ & $\begin{array}{lll}1 & 4 & 3\end{array}$ \\
\hline$F_{7}(x)=x^{6}+5 x^{4}+6 x^{2}+1$ & $\begin{array}{lll}1 & 5 & 6\end{array}$ \\
\hline$F_{8}(x)=x^{7}+6 x^{5}+10 x^{3}+4 x$ & $\begin{array}{llll}1 & 6 & 10 & 4 \\
\end{array}$ \\
\hline . & $\vdots$ \\
\hline
\end{tabular}

The first few Fibonacci polynomials and their coefficient array are shown as follows:

Table 1. The first few Fibonacci polynomials and the array of their coefficients

Considering the rule of formation of Fibonacci polynomials, we write the polynomials in descending order. We get the coefficient of the $k$-th term of $F_{n}(x)$ by adding the coefficient of the $k$-th term of $F_{n-1}(x)$ and the $(k-1)$-st term of $F_{n-2}(x)$. Hence, the array of coefficients formed has the same rule of formation as Pascal's triangle when it is written in left-justified form, except that each column is moved one line lower. Therefore, the coefficients formed are those elements that appear along the diagonals formed by beginning in the left-most column and preceding up one and right one throughout the left-justified Pascal triangle [12].

Definition 2.1. [9] Let $n$ and $k \neq 0$ be natural numbers, then there exist unique numbers $m$ and $r$ such that $n=m k+r(0 \leq r<k)$. The generalized $k$-Fibonacci numbers $F_{n}^{(k)}$ are defined by

$$
F_{n}^{(k)}=\frac{1}{(\sqrt{5})^{k}}\left(\alpha^{m+2}-\beta^{m+2}\right)^{r}\left(\alpha^{m+1}-\beta^{m+1}\right)^{k-r}, n=m k+r
$$


where $\alpha=(1+\sqrt{5}) / 2$ and $\beta=(1-\sqrt{5}) / 2$.

The first numbers of the family for $k=2$ is as follows:

$$
\left\{F_{n}^{(2)}\right\}=\{1,1,1,2,4,6,9,15,25,40,64, \ldots\} .
$$

It is well known that the relation of generalized $k$-Fibonacci and Fibonacci numbers is

$$
F_{n}^{(k)}=\left(F_{m}\right)^{k-r}\left(F_{m+1}\right)^{r}
$$

where $n=m k+r$. When $k=1$ in the last equation, we get that $m=n$ and $r=0$. Therefore, $F_{n}^{(1)}=F_{n}$.

\section{Main results}

Definition 3.1. The 2-Fibonacci polynomials are defined by

$$
F_{n+1}^{(2)}(x)=x F_{n}^{(2)}(x)+F_{n-1}^{(2)}(x), \quad n \geq 1,
$$

where $F_{0}^{(2)}(x)=1$ and $F_{1}^{(2)}(x)=1$.

\begin{tabular}{|c|c|c|c|c|c|c|c|c|c|}
\hline 2-Fibonacci polynomials & \multicolumn{9}{|c|}{ Coefficients array } \\
\hline$F_{1}^{(2)}(x)=1$ & 1 & & & & & & & & \\
\hline$F_{2}^{(2)}(x)=1+x$ & 1 & 1 & & & & & & & \\
\hline$F_{3}^{(2)}(x)=1+x+x^{2}$ & 1 & 1 & 1 & & & & & & \\
\hline$F_{4}^{(2)}(x)=1+2 x+x^{2}+x^{3}$ & 1 & 2 & 1 & 1 & & & & & \\
\hline$F_{5}^{(2)}(x)=1+2 x+3 x^{2}+x^{3}+x^{4}$ & 1 & 2 & 3 & 1 & 1 & & & & \\
\hline$F_{6}^{(2)}(x)=1+3 x+3 x^{2}+4 x^{3}+x^{4}+x^{5}$ & 1 & 3 & 3 & 4 & 1 & 1 & & & \\
\hline$F_{7}^{(2)}(x)=1+3 x+6 x^{2}+4 x^{3}+5 x^{4}+x^{5}+x^{6}$ & 1 & 3 & 6 & 4 & 5 & 1 & 1 & & \\
\hline$F_{8}^{(2)}(x)=1+4 x+6 x^{2}+10 x^{3}+5 x^{4}+6 x^{5}+x^{6}+x^{7}$ & 1 & 4 & 6 & 10 & 5 & 6 & 1 & 1 & \\
\hline$F_{9}^{(2)}(x)=1+4 x+10 x^{2}+10 x^{3}+15 x^{4}+6 x^{5}+7 x^{6}+x^{7}+x^{8}$ & 1 & 4 & 10 & 10 & 15 & 6 & 7 & 1 & 1 \\
\hline
\end{tabular}

The first few 2-Fibonacci polynomials are displayed below as well as the array of their coefficients.

Table 2. The first few 2-Fibonacci polynomials and the arrays of their coefficients

Considering the rule of formation of the 2-Fibonacci polynomials, we write the polynomials in ascending order. In order to obtain the coefficient of the $k$-th term of $F_{n}^{(2)}(x)$, we add the coefficients of the $(k-1)$-st term of $F_{n-1}^{(2)}(x)$ and $k$-th term of $F_{n-2}^{(2)}(x)$. The array of formed coefficients has the same rule of formation as Pascal's triangle when it is written in left-justified form, except that each line is written two times and each column is moved one line upper, if we use the formation of the 2-Fibonacci polynomials and write the polynomials in ascending order. Therefore, the coefficients appear along the diagonals formed by beginning in the right-most column and preceding up one and left one throughout the left-justified Pascal triangle whose each line is written two times, e.g., fourth and fifth lines of Pascal's triangle are written bold. 
Theorem 3.2. The 2-Fibonacci polynomials are given by

$$
F_{n}^{(2)}(x)=F_{n-1}(x)+F_{n}(x), \quad n \geq 1 .
$$

Proof. For $n=1$, the claim is true, since

$$
F_{1}^{(2)}(x)=1=F_{0}(x)+F_{1}(x) .
$$

Suppose that it is true for $n=k$, that is

$$
F_{k}^{(2)}(x)=F_{k-1}(x)+F_{k}(x) .
$$

We should show that the claim is true for $n=k+1$. We have

$$
\begin{aligned}
F_{k+1}^{(2)}(x) & =x F_{k}^{(2)}(x)+F_{k-1}^{(2)}(x) \\
& =x\left(F_{k-1}(x)+F_{k}(x)\right)+F_{k-2}(x)+F_{k-1}(x) \\
& =x F_{k-1}(x)+F_{k-2}(x)+x F_{k}(x) F_{k-1}(x) \\
& =F_{k}(x)+F_{k+1}(x) .
\end{aligned}
$$

We find Binet's formulas for the 2-Fibonacci polynomials. Let $\alpha(x)$ and $\beta(x)$ be the solutions of the quadratic equation $t^{2}-x t-1=0$ :

$$
\alpha(x)=\frac{x+\sqrt{x^{2}+4}}{2} \text { and } \beta(x)=\frac{x-\sqrt{x^{2}+4}}{2}
$$

Notice that $\alpha(1)=\alpha$ and $\beta(1)=\beta ; \alpha(2)=1+\sqrt{2}$ and $\beta(2)=1-\sqrt{2}$ are roots of the characteristic equation $x^{2}-2 x-1=0$ of the Pell recurrence relation. We can verify that

$$
F_{n+1}^{(2)}(x)=\frac{\alpha^{n}(x)(\alpha(x)+1)-\beta^{n}(x)(\beta(x)+1)}{\alpha(x)-\beta(x)} .
$$

The 2-Fibonacci polynomials are obtained by the matrix $Q_{2}=\left(\begin{array}{cc}x & 1 \\ 1 & 0\end{array}\right)$,

$$
\begin{aligned}
Q_{2}^{n-1}+Q_{2}^{n-2} & =\left(\begin{array}{cc}
x & 1 \\
1 & 0
\end{array}\right)^{n-1}+\left(\begin{array}{ll}
x & 1 \\
1 & 0
\end{array}\right)^{n-2} \\
& =\left(\begin{array}{cc}
F_{n}^{(2)}(x) & F_{n-1}^{(2)}(x) \\
F_{n-1}^{(2)}(x) & F_{n-2}^{(2)}(x)
\end{array}\right),
\end{aligned}
$$

can be shown by mathematical induction.

We can write the following interesting determinant identity

$$
\operatorname{det}\left(\begin{array}{cc}
F_{n}^{(2)}(x) & F_{n-1}^{(2)}(x) \\
F_{n-1}^{(2)}(x) & F_{n-2}^{(2)}(x)
\end{array}\right)=(-1)^{n} x .
$$

Theorem 3.3.

$$
F_{n+1}^{(2)}(x)=1+x \sum_{i=1}^{n} F_{i}(x)
$$


Proof. Using the recurrence relation $F_{n}(x)=x F_{n-1}(x)+F_{n-2}(x)$,

$$
\sum_{1}^{n} F_{i+1}(x)=x \sum_{1}^{n} F_{i}(x)+\sum_{1}^{n} F_{i-1}(x) .
$$

That is

$$
\begin{aligned}
F_{n+1}^{(2)}(x) & =F_{n+1}(x)+F_{n}(x) \\
& =x \sum_{1}^{n} F_{i}(x)+F_{0}(x)+F_{1}(x) .
\end{aligned}
$$

Since $F_{0}(x)=0$, it follows that

$$
\begin{aligned}
x \sum_{1}^{n} F_{i}(x) & =F_{n+1}(x)+F_{n}(x)-1 \\
F_{n+1}^{(2)}(x) & =1+x \sum_{1}^{n} F_{i}(x) .
\end{aligned}
$$

Theorem 3.4. More generally, the sum of the elements along the diagonal beginning at row $n$ is $F_{n}^{(2)}(x)$; that is,

$$
F_{n}^{(2)}(x)=\sum_{j=0}^{\left[\frac{n-1}{2}\right]}\left(\begin{array}{c}
n-j-1 \\
j
\end{array}\right) x^{n-2 j-1}+\left(\begin{array}{c}
n-j-2 \\
j
\end{array}\right) x^{n-2 j-2} .
$$

Proof. We have

$$
\frac{y}{1-x y-y^{2}}=\sum_{n=0}^{\infty} F_{n}(x) y^{n}
$$

But

$$
\begin{aligned}
\frac{1}{1-2 t z+z^{2}} & =\sum_{n=0}^{\infty}\left[\sum_{j=0}^{\left[\frac{n-1}{2}\right]}(-1)^{j}\left(\begin{array}{c}
n-j \\
j
\end{array}\right)(2 t)^{n-2 j}\right] z^{n} \\
U_{n}(t) & =\sum_{j=0}^{\left[\frac{n-1}{2}\right]}(-1)^{j}\left(\begin{array}{c}
n-j \\
j
\end{array}\right)(2 t)^{n-2 j} .
\end{aligned}
$$

Let $z=i y$ and $t=\frac{x}{2 i}$, where $i^{2}=-1$, then

$$
\frac{1}{1-x y-y^{2}}=\sum_{n=0}^{\infty} i^{n} U_{n}\left(\frac{x}{2 i}\right) y^{n}
$$

Multiplying the last equation by $y$, we get

$$
\frac{y}{1-x y-y^{2}}=\sum_{n=0}^{\infty} i^{n} U_{n}\left(\frac{x}{2 i}\right) y^{n+1} \text {. }
$$


It follows that

$$
\begin{aligned}
F_{n}(x) & =i^{n} U_{n}(x / 2 i) \\
& =i^{n} \sum_{j=0}^{\left[\frac{n}{2}\right]}(-1)^{j}\left(\begin{array}{c}
n-j-1 \\
j
\end{array}\right)(x / i)^{n-2 j-1} \\
& =\sum_{j=0}^{\left[\frac{n}{2}\right]}\left(\begin{array}{c}
n-j-1 \\
j
\end{array}\right) x^{n-2 j-1} . \\
F_{n-1}(x) & =\sum_{j=0}^{\left[\frac{n-1}{2}\right]}\left(\begin{array}{c}
n-j-2 \\
j
\end{array}\right) x^{n-2 j-2} . \\
F_{n}^{(2)}(x) & =F_{n}(x)+F_{n-1}(x) \\
& =\sum_{j=0}^{\left[\frac{n-1}{2}\right]}\left(\begin{array}{c}
n-j-1 \\
j
\end{array}\right) x^{n-2 j-1}+\left(\begin{array}{c}
n-j-2 \\
j
\end{array}\right) x^{n-2 j-2} .
\end{aligned}
$$

Theorem 3.5. The derivative of the 2-Fibonacci polynomial $F_{n}^{(2)}(x)$ is

$$
\left(F_{n}^{(2)}(x)\right)^{\prime}=\sum_{i=1}^{n-1} F_{i}(x) \cdot\left(F_{n-i}(x)+F_{n-i-1}(x)\right) .
$$

Proof. It is known that

$$
\begin{aligned}
F_{n}^{(2)}(x) & =F_{n}(x)+F_{n-1}(x) \\
\left(F_{n}^{(2)}(x)\right)^{\prime} & =\left(F_{n}(x)+F_{n-1}(x)\right)^{\prime} \\
\left(F_{n}(x)\right)^{\prime} & =\sum_{i=1}^{n} F_{i}(x) F_{n-i}(x) \\
\left(F_{n-1}(x)\right)^{\prime} & =\sum_{i=1}^{n-1} F_{i}(x) F_{n-i-1}(x) \\
\left(F_{n}^{(2)}(x)\right)^{\prime}= & \sum_{i=1}^{n-1} F_{i}(x)\left(F_{n-i}(x)+F_{n-i-1}(x)\right) .
\end{aligned}
$$

Example 3.6. The derivative of

$$
F_{5}^{(2)}(x)=x^{4}+x^{3}+3 x^{2}+2 x+1
$$

is

$$
\begin{aligned}
\sum_{i=1}^{4} F_{i}(x)\left[F_{5-i}(x)+F_{5-i-1}(x)\right] & =F_{1}(x)\left[F_{4}(x)+F_{3}(x)\right] \\
& +F_{2}(x)\left[F_{3}(x)+F_{2}(x)\right] \\
& +F_{3}(x)\left[F_{2}(x)+F_{1}(x)\right] \\
& +F_{4}(x)\left[F_{1}(x)+F_{0}(x)\right]
\end{aligned}
$$




$$
\begin{aligned}
& =F_{1}(x) F_{4}(x)+F_{1}(x) F_{3}(x) \\
& +F_{2}(x) F_{3}(x)+F_{2}(x) F_{2}(x) \\
& +F_{3}(x) F_{2}(x)+F_{3}(x) F_{1}(x) \\
& +F_{4}(x) F_{1}(x)+F_{4}(x) F_{0}(x) \\
& =2 F_{1}(x) F_{4}(x)+2 F_{1}(x) F_{3}(x) \\
& +2 F_{2}(x) F_{3}(x)+F_{2}^{2}(x) \\
& =2 \cdot 1 \cdot\left(x^{3}+2 x\right)+2 \cdot 1 \cdot\left(x^{2}+1\right) \\
& +2 \cdot x \cdot\left(x^{2}+1\right)+x^{2} \\
& =4 x^{3}+3 x^{2}+6 x+2 .
\end{aligned}
$$

\section{Conclusion}

In the present paper, we define 2-Fibonacci polynomials and give some identities between the 2-Fibonacci polynomials and Fibonacci polynomials. Also, we present some properties of the 2-Fibonacci polynomials. We introduce the derivative of the derivatives of the 2-Fibonacci polynomials. This enables us to give in a straightforward way several formulas for the sums of such polynomials. These identities can be used to develop new identities of polynomials.

\section{References}

[1] Berg, C. (2011) Fibonacci numbers and orthogonal polynomials, Arab Journal of Mathematical Sciences, 17, 75-88.

[2] Falcon S. \& Plaza, A. (2009) On $k$-Fibonacci sequences and polynomials and their derivatives, Chaos, Solutions and Fractals, 39, 1005-1019.

[3] Hoggatt, Jr. V. E. \& Lind, D. A. (1968) Symbolic Substitutions in to Fibonacci Polynomials, The Fibonacci Quarterly, 6(5), 55-74.

[4] Hoggatt, Jr. V. E., Leonard, H. T. Jr. \& Philips, J. W. (1971) Twenty-four Master Identities, The Fibonacci Quarterly, 9(1), 1-17.

[5] Hoggatt, Jr. V. E. \& Bicknell, M. (1973) Generalized Fibonacci polynomials, Fibonacci Quarterly, 11(5), 457-465.

[6] Ivie, J. (1972) A General Q-Matrix, Fibonacci Quarterly, 10(3), 255-261.

[7] Koshy, T. (2001) Fibonacci and Lucas Numbers with Applications, John Wiley \& Sons, Inc., Canada.

[8] Lee G. Y. \& Asci, M. (2012) Some Properties of the $(p, q)$-Fibonacci and $(p, q)$-Lucas Polynomials, Journal of Applied Mathematics, 2012, 18 pages. 
[9] Mikkawy M. \& Sogabe, T. (2010) A new family of $k$-Fibonacci numbers, Applied Mathematics and Computation, 215, 4456-4461.

[10] Nalli, A. \& Haukkanen, P. (2009) On generalized Fibonacci and Lucas polynomials, Chaos, Solitons and Fractals, 42(5), 3179-3186.

[11] Panwar, Y. K., Singh, B. \& Gupta, V. K. (2013) Generalized Fibonacci Polynomials, Turkish Journal of Analysis and Number Theory, 1(1), 43-47.

[12] Ramirez, J. (2014) On convelved generalized Fibonacci and Lucas polynomials, Applied Mathematics and Computation, 229, 208-213.

[13] Swamy, M. N. S. (1965) Problem B-74, The Fibonacci Quarterly, 3(3), 236.

[14] Tuğlu, N., Koçer, E. G. \& Stakhov, A. (2011) Bivariate fibonacci like $p$-polynomials, Applied Mathematics and Computation, 217, 10239-10246.

[15] Ye, X. \& Zang, Z. (2017) A common generalization of convolved generalized Fibonacci and Lucas polynomials and its applications, Applied Mathematics and Computation, 306, $31-37$. 